\title{
Do male and female trauma patients receive the same prehospital care?: an observational follow-up study
}

\author{
Rebecka Rubenson Wahlin ${ }^{1,5^{*}}$, Sari Ponzer ${ }^{1}$, Hanna Lövbrand ${ }^{1}$, Markus Skrivfars ${ }^{2}$, Hans Morten Lossius ${ }^{1,3}$ \\ and Maaret Castrén ${ }^{1,4}$
}

\begin{abstract}
Background: Trauma-related mortality can be lowered by efficient prehospital care. Less is known about whether gender influences the prehospital trauma care provided. The aim of this study was to explore gender-related differences in prehospital trauma care of severely injured trauma patients, with a special focus on triage, transportation, and interventions.

Methods: We performed a retrospective observational study based on local trauma registries and hospital and ambulance records in Stockholm County, Sweden. A total of 383 trauma patients (279 males and 104 females) $>15$ years of age with an Injury Severity Score (ISS) of $>15$ transported to emergency care hospitals in the Stockholm area were included.

Results: Male patients had a 2.75 higher odds ratio ( $95 \%$ Cl, 1.2-6.2) for receiving the highest prehospital priority compared to females on controlling for injury mechanism and vital signs on scene. No significant difference between genders was detected regarding other aspects of the prehospital care provided.

Conclusions: This study indicated that prehospital prioritization among severely injured late adolescent and adult trauma patients differs between genders. Knowledge of a more diffuse presentation of symptoms in female trauma patients despite severe injury may help to adapt and improve prehospital trauma care for this group.
\end{abstract}

Keywords: Gender, Injury, Late adolescent and adult trauma care, Prehospital care, Emergency services

\section{Background}

Trauma is a major cause of death and permanent disability worldwide [1]. Most trauma-related deaths occur on the scene of the trauma. In European countries, nine prehospital deaths occur for each hospital death of trauma patients aged 65 and younger [2]. Preventable trauma deaths are frequently caused by hemorrhagic shock, a reversible state if treated in time [3]. Prehospital hemorrhage control and initiation of fluid therapy, as well as preventing hypothermia and managing airways, may improve survival $[3,4]$.

\footnotetext{
* Correspondence: rebecka.rubenson.wahlin@ki.se

'Department of Clinical Science and Education, Södersjukhuset, Karolinska Institutet, SE-118 83 Stockholm, Sweden

${ }^{5}$ Department of Anesthesia and Intensive Care, Södersjukhuset, SE-118 83

Stockholm, Sweden

Full list of author information is available at the end of the article
}

Some studies have suggested differences between genders in terms of type and severity of trauma, the prehospital care provided, and outcome. Wohltmann and colleagues showed that young males have a $27 \%$ higher risk of dying from trauma compared to females [5]. These findings were supported by a Swedish study reporting males to have an increased risk of 1-year mortality even when adjusted for injury severity and other probable confounders [6]. Correct triage and direct transport to a trauma center has been shown to be associated with improved survival [7]. Gomez et al. reported that severely injured females are less likely to be directed to a trauma center [8].

Research on prehospital general trauma care in terms of assessment and treatment on scene and during transportation to hospital is scarce. As far as we know, no studies have been published directly focusing on gender 
differences of the severely injured trauma patient, although gender aspects have been mentioned. Some studies have been conducted regarding other aspects of the prehospital care. Meisel et al. studied prehospital care of patients presenting with acute chest pain and found that females were less likely to receive aspirin, nitroglycerin, and intravenous access compared to their male counterparts [9]. Furthermore, Kaul et al. reported that women presenting at an emergency department with coronary syndromes were less likely than men to be admitted to an acute care hospital and to be treated with coronary revascularization procedures [10]. In order to ensure gender-equal prehospital care, gender aspects should be integrated in future research $[11,12]$.

The aim of this study was to explore gender-related differences in prehospital trauma care of severely injured trauma patients, with a special focus on triage, transportation, and interventions.

\section{Methods}

\section{Study setting}

This study was conducted in the area of the Stockholm County Council (SCC), consisting of 26 municipalities covering $6519 \mathrm{~km}^{2}$ and including an archipelago of approximately 30,000 islands. Stockholm County has about two million inhabitants, which is about $20 \%$ of the Swedish population [13]. The SCC has the overall responsibility for all healthcare, including the emergency medical services (EMS) and the seven emergency care hospitals, one of which has two sites, but only one of them can be regarded as a level-1 trauma center according to the American College of Surgeons' criteria [14]. Two private organizations run the prehospital EMS on contracts with the SCC, as well as one organization run by the SCC itself. At the time of the data collection (2008), there were 55 ground ambulances, one helicopter, one mobile intensive care unit (MICU), and three rapidresponse vehicles operating in the area. One of the rapidresponse vehicles was staffed by an anesthesiologist and the other two by a nurse anesthetist, as well as emergency medical technicians (EMTs). The MICU was staffed by one ambulance nurse and an EMT. The rapid-response vehicle was called in, in addition to a regular ambulance, for severe accidents with the purpose of starting early advanced resuscitation. The helicopter was staffed by a nurse anesthetist, and all regular ground ambulances by EMTs and registered nurses.

The EMS uses three different levels of priority when transporting patients to hospital, with priority 1 being the highest and most urgent level and priority 3 being only transportation. The priority of severe traumas is based on a trauma triage and transport protocol (implemented in 2007) for prehospital use [15] and is derived from ACS-COT field triage criteria [16]. The trauma triage protocol includes vital parameters (i.e., SBP $<90$, $\mathrm{RR}<10$ or $>29$, and GCS $<14$ ), anatomical injuries, and trauma mechanism. The triage protocol states that if the trauma patient fulfills any of the triage crieria he/she should be transported directly to the trauma center even if it means bypassing a nearer hospital (Fig. 1). Patients who meet the criteria for transport to the trauma center are assigned a level 1 transport priority (i.e., the highest level of prehospital priority) [15].

\section{Study population}

Included were adult and late adolescent trauma patients ( $>15$ years of age) with an Injury Severity Score (ISS) $>15$, transported by ground ambulance or helicopter to any of the seven hospitals providing emergency care in the Stockholm area during the period January 1st - December 31st, 2008. Patients with cardiac arrest due to trauma and ongoing cardiopulmonary resuscitation (CPR) during transport to hospital were included even if no return of spontaneous circulation (ROSC) had occurred during transport. Patients declared dead on scene due to trauma and for whom no resuscitative measures were taken, patients admitted to the reporting hospital $>24 \mathrm{~h}$ after the trauma, and patients suffering from asphyxia due to drowning were excluded. In addition, we excluded patients transported from another county for specialist care and/or transfers after $>24 \mathrm{~h}$ to the university hospital after admission to any of the other hospitals.

Variables recorded were age, gender, predominant type of injury, mechanism of injury, ICD-10 diagnosis, intentional injury, prehospital cardiac arrest, prehospital time intervals, prehospital competence level, i.e., basic (EMT and nurse) or advanced (nurse anesthetist or anesthesiologist), type of prehospital transportation, airway management, hospital length of stay (LOS), and 30day mortality, all in accordance with the Utstein trauma template [17]. In addition, the following variables were added for the purpose of this study: prehospital priority (priority 1/other), transport to trauma center (yes/no), administered fluid and analgesics, ISS [18], and the Revised Trauma Score (RTS) [19], including the Glasgow Coma Scale (GCS) [20], systolic blood pressure, respiratory rate, and the 24-h mortality.

Primary outcome measures were prehospital priority and prehospital analgesics given. Prehospital priority was chosen since this measure was considered to be a result of the overall prehospital assessment of the patient, in terms of both triage and transport decision. The assessment was based on the trauma triage and transport protocols used in our system (Fig. 1). Prehospital analgesics given were chosen as a measure of the prehospital care as seen from a patient perspective.

Secondary outcomes were transport to trauma center, competence level of the prehospital staff, type of prehospital 


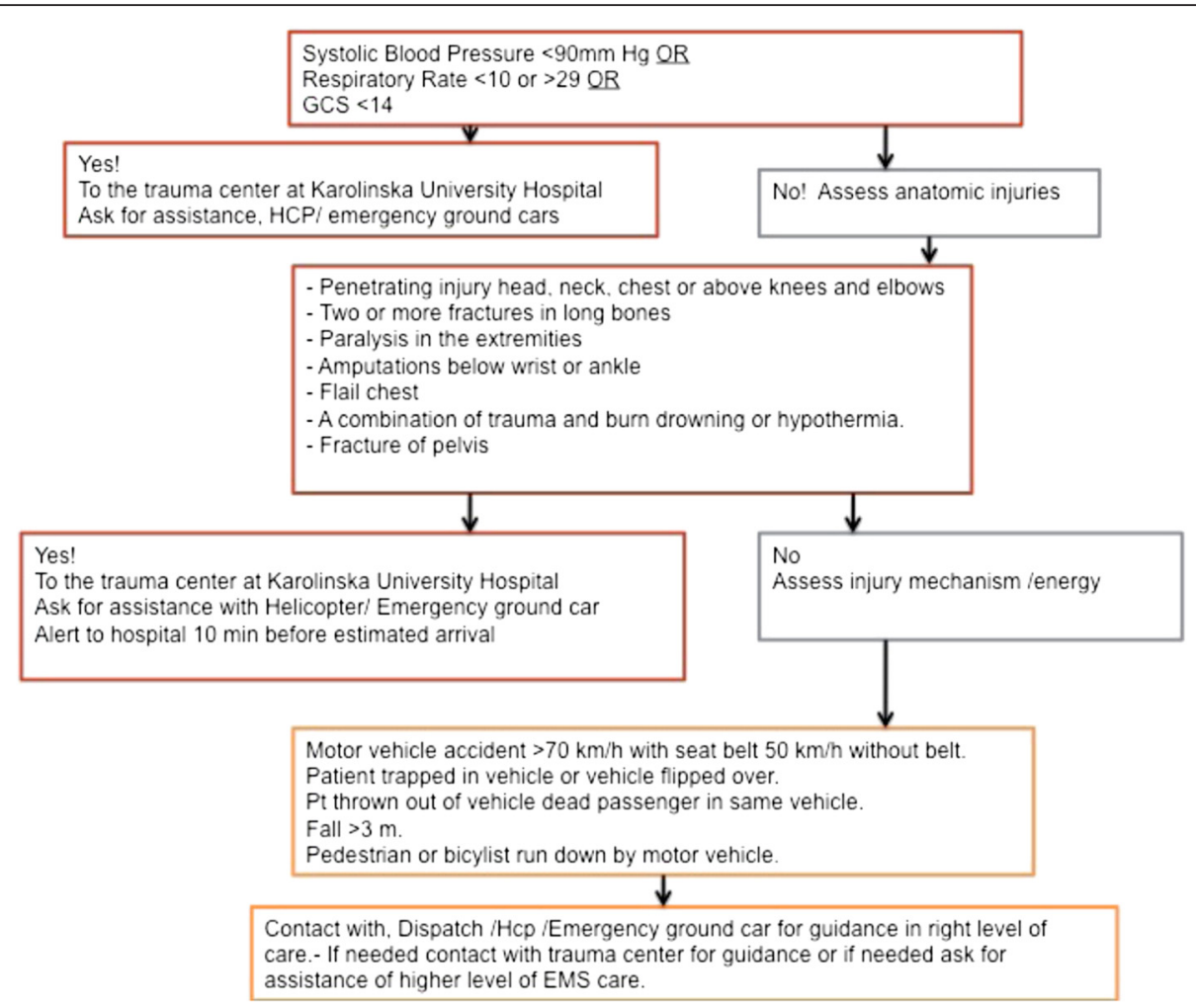

Fig. 1 Trauma triage protocol. The trauma triage protocol used in the SCC. The triage protocol also states where to transport the patient. When a patient meets the criteria for transport to a trauma center, the priority is automatically priority 1

transportation, prehospital airway management, prehospital fluids, prehospital immobilization, 30-day mortality, 24-h mortality, hospital LOS, total prehospital time, and prehospital on-scene time. These outcomes were chosen in order to get a broader view of both the system and the prehospital care.

\section{Data collection}

Data were collected from the trauma registries at Karolinska University Hospital (two sites) (KVITTRA/ QUITC, version 14.0) and from Södersjukhuset, a large teaching hospital (TRAUMAREG version TraumaSys 2000-2001, version 1.1.). For Södersjukhuset, the trauma registry data regarding length of stay (LOS) were completed via the hospital's inpatient digital registration system (Pasett-DRG, version 1.61). Pre-hospital data stemmed from digital ambulance records (CAK-net) used by all ambulance caregivers.

Patients from the four hospitals without trauma registries were identified by a manual search of admission records for each emergency department. Records for all patients with any type of trauma transported by ambulance or helicopter to surgical or orthopedic sections of the emergency departments (EDs), all patients with an ED priority level of 1 or 2 (urgent triage levels), and/or all of those admitted to a hospital ward from the ED were examined. In addition, records of all patients with suspected head trauma or patients directly admitted to the ICU or operating room from the ED were examined regardless of the priority given at the ED. It was not possible to obtain hospital admission records for one of the minor hospitals without trauma registries, and therefore only records for patients reported as "pre-alert" trauma patients were scanned. All patients were identified through the unique personal social security number given to every Swedish citizen. Foreign patients receive a temporary identification number given by the admitting hospital and therefore it was also possible to track these patients. Inpatient data were retrieved via the hospitals' digital records (Take Care, Melior, and Cambio Cosmic).

The Abbreviated Injury Score (AIS, version 2005) and Injury Severity Score (ISS) [18] were calculated by a trained trauma registrar and by one of the authors (RRW).

\section{Ethical approval}

The study received ethical approval from the Regional Ethical Review Board in Stockholm (Reg. Nos.: 2007/ 1113-31, 2010/1979-32, 2013/1718-32, and 2014/691-32).

\section{Statistics}

Since none of the background variables showed a normal distribution, medians and interquartile ranges (IQRs) 
were calculated for continuous variables and, for categorical variables, counts (n) and percent (\%) were used. The Mann-Whitney $U$-test was used to calculate continuous data and chi-square for categorical data.

Differences between genders regarding prehospital priority and care were first analyzed using univariable logistic regression. Thereafter, adjusted odds ratios (ORs) with $95 \%$ confidence intervals (CIs) for primary outcomes were derived by multivariable logistic regression analysis, and, for both models, females were used as the reference group. The covariates included in both the univariable and multivariable models were age, predominant type of injury, intentional injury, injury mechanisms, prehospital cardiac arrest, RTS category of the Glasgow Coma Scale, systolic blood pressure, and respiratory rate. Separate analyses of regression models were performed by stratification of the covariates in order to evaluate whether or not gender-based differences were affected by patient or injury characteristics. In these models, each stratification variable was excluded from its respective model. The data analyses followed a similar methodology to that employed by Gomez et al. 2012 in their study on gender-related differences in access to trauma center care [8]. Model calibration was estimated using the Hosmer-Lemeshow statistic and discrimination using the c-statistic. In all models, the c-statistic exceeded 0.85 , suggesting excellent discrimination, and the models showed adequate calibration. The software IBM SPSS Statistics, version 22.0.0.0, was used for the analysis. The statistical significance level was set to $p<0.05$.

\section{Results}

\section{Background data}

During the study period, a total of 383 patients, 279 males $(72.8 \%)$ and 104 females $(27.2 \%)(p<0.001)$, with an ISS $>15$ were included. Table 1 shows the baseline characteristics for all patients. There were no significant differences in the median age between males (median 45 years, IQR 27-64) and females (median 50 years, IQR 29-77) (Table 1).

The median ISS did not differ between males (24, IQR 18-30) and females (25, IQR 18-30) (Table 1). The most frequent injury type for both genders was blunt trauma and the predominant injury mechanism was a traffic accident (Table 1). The anatomical injuries did not differ between groups and for both genders the most frequent injury was head injury (Table 1). Female gender was significantly more frequent among patients with self-inflicted injuries, while males were more often exposed to assaults $(p=0.041)$. RTS categories (Glasgow Coma Scale, systolic blood pressure, and respiratory rate) did not differ between genders and neither did the rate of prehospital cardiac arrest (Table 1).

\section{Outcome data}

There was no difference between genders regarding prehospital on-scene time (Table 2). Male patients were significantly more often given priority $1 \quad(p<0.001)$, were more often transported straight to a trauma center $(p=$ $0.016)$ and were also more often allocated the highest level of prehospital competence $(p=0.033)$ compared to female trauma patients. Type of transportation, prehospital airway management, fluids, analgesics, or immobilization did not differ between genders. The same was true for hospital LOS, as well as for mortality at $24 \mathrm{~h}$ or at 30 days (Table 2 ).

\section{Injury mechanism within the blunt trauma group}

Blunt trauma was the most common injury type (Table 1) for both genders. Within the blunt trauma group, the most common trauma mechanism was traffic-related injury for both genders, but the second most common mechanism differed between genders. For women, it was low-energy falls and, for men high-energy falls ( $p=$ $0.019)$. On stratifying age and trauma mechanism, the most common injury mechanism in the age group $\geq 65$ was low energy falls for both genders. Low energy falls accounted for $77.8 \%$ of the cases involving women 65 years of age or older and, among men in the same age group, low-energy falls accounted for $66.7 \%$ of the trauma cases (Table 3).

\section{Logistic regression analyses}

The univariable logistic regression analysis showed an OR of 2.89 (95\% CI, 1.6-5.1; $p<0.001$ ) for male patients to receive the highest priority, compared to females. After adjusting for age, predominant type of injury, intentional injury, injury mechanisms, prehospital cardiac arrest, and RTS, the OR was 2.75 (95\% CI, 1.2-6.2; $p=0.015$ ). No interactions were found between the patients' gender and the variables adjusted for.

When the analyses were stratified and adjusted for the association between the highest prehospital priority and male gender, the likelihood of a higher priority was relatively the same over strata (Fig. 2). The exceptions were the injury mechanism categories, "Low-energy fall", OR 5.12 (95 \% CI, 1.1-23.4) and "Other", OR 9.05 (95\% CI, 0.44-187.9), which showed an increased likelihood of a higher priority for males. In addition, the injury mechanism "high-energy fall" showed a lower likelihood OR of 1.27 (95\% CI, $0.21-7.62$ ) of receiving the highest priority for males.

There was no difference between genders regarding prehospital-administered analgesics $(31.5 \%$ and $28.8 \%$, respectively; $p=0.611$ ) and the multivariable logistic regression analysis did not show any significant gender differences regarding the likelihood of either gender to receive analgesics $\left(\mathrm{OR}_{\text {adj }}\right.$ for males, 0.86 ; $95 \% \mathrm{CI}, 0.49-1.51$; $p=0.609$ ). However, there was an increased likelihood for 
Table 1 Patient characteristics and background factors by gender $(n=383)$

\begin{tabular}{llll}
\hline Gender & $\begin{array}{l}\text { Male } \\
n(\%)\end{array}$ & $\begin{array}{l}\text { Female } \\
n(\%)\end{array}$ & $\begin{array}{l}P \text { value } \\
* \text { sign. }\end{array}$ \\
\hline Patients & $279(72.8)$ & $104(27.2)$ & $<0.001^{*}$ \\
Age groups & & & 0.050 \\
$\quad$ 15-39 years & $116(41.6)$ & $37(35.6)$ & \\
40-64 years & $96(34.4)$ & $29(27.9)$ & \\
$\geq 65$ years & $67(24.0)$ & $38(36.5)$ &
\end{tabular}

Injury Severity Score (ISS),

ISS, 15-29

ISS, 30-44

ISS $\geq 45$

Predominant type of injury

Blunt
Penetrating
njury mechanism
Traffic
Low-energy fall
High-energy fall
Other

$252(90.3) \quad 98(94.2)$

$55(19.7) \quad 10(9.6)$

Intentional injury

Accident
Self-inflicted
Assault
Missing

Predominant Anatomical Injury

Isolated head

Head

Chest

Abdomen

Pelvis

Spine and Spinal cord

Amputated limb or severe

injured extremity

$\geq 2$ long bone fractures

Missing

Systolic blood pressure, RTS category

Systolic blood pressure, RTS 4

Systolic blood pressure, RTS 0-3

Missing

Respiratory rate, RTS category,

Respiratory rate, RTS 4

Respiratory rate, RTS 0-3

Missing
209 (74.9) $76(73.1)$

$50(17.9) \quad 20(19.2)$

$20(7.2) \quad 8(7.7)$

$27(9.7) \quad 6(5.8)$

$113(40.6) \quad 39(37.5)$

$43(15.5) \quad 28(26.9)$

$68(24.5) \quad 27(26)$

0.935

0.225

$0.019^{*}$

$229(82.7) \quad 87(84.5)$

$13(4.7) \quad 10(9.7)$

$35(12.6) \quad 6(5.8)$

21

54 (19.6) $\quad 23(22.3)$

$74(26.8) \quad 32(31.1)$

$69(25.0) \quad 20(19.4)$

$35(12.7) \quad 8(7.8)$

10 (3.6) $\quad 12(11.7)$

$22(8.0) \quad 5(5.9)$

$6(2.2) \quad 1(1.0)$

$6(2.2) \quad 2(1.9)$

31

$226(85.0) \quad 84(84.0)$

$40(15.0) \quad 16(16.0)$

$13 \quad 4$

$226(85.6) \quad 85(82.5)$

$38(14.4) \quad 14(14.1)$

$15 \quad 5$

$0.041^{*}$

0.065

0.951
Table 1 Patient characteristics and background factors by gender $(n=383)$ (Continued)

\begin{tabular}{clll}
\hline Glasgow Coma Scale, RTS category & & 0.200 \\
Glasgow Coma Scale, RTS 3-4 & $207(76.4)$ & $85(82.5)$ & \\
Glasgow Coma Scale, RTS 0-2 & $64(23.6)$ & $18(17.5)$ & \\
Prehospital cardiac arrest & $12(4.3)$ & $3(2.9)$ & 0.538 \\
\hline
\end{tabular}

* is a marker for a significant finding and the $p$ level was set to $<0,05$

Table 2 Outcome variables by gender

\begin{tabular}{llll}
\hline Gender & $\begin{array}{l}\text { Male } \\
n(\%)\end{array}$ & $\begin{array}{l}\text { Female } \\
n(\%)\end{array}$ & $\begin{array}{l}P \text { value } \\
{ }^{*} \text { sign. }\end{array}$ \\
\hline $\begin{array}{llll}\text { Prehospital priority } \\
\quad \text { Priority } 1\end{array}$ & $238(88.1)$ & $72(72.0)$ & $<0.001^{*}$ \\
$\quad$ Priority $>1$ & $32(11.9)$ & $28(28.0)$ & \\
$\begin{array}{l}\text { Transport to trauma center } \\
\quad \text { Yes }\end{array}$ & $232(83.2)$ & $75(72.1)$ & \\
No & $47(16.8)$ & $29(27.9)$ &
\end{tabular}

Highest level of prehospital

$0.033^{*}$ competence

$\begin{array}{lll}\text { Basic } & 105(38.7) & 52(51.0 \\ \text { Advanced } & 166(61.3) & 50(49.0)\end{array}$

Type of prehospital transportation

Ground ambulance

208(76.8) 76(73.1)

Helicopter

63(23.2) 28(26.9)

Prehospital airway management

Not intubated

$248(89.9) \quad 92(91.1)$

Intubated

$28(10.1) \quad 9(8.9)$

Prehospital fluids

No fluids

$177(63.4) \quad 76(73.1)$

Fluids

$102(36.6) \quad 28(26.9)$

Prehospital analgesics

No analgesics

Analgesics

Prehospital immobilization of neck and spine

No immobilization of both neck and spine

Immobilization of both

neck and spine

30-day mortality (yes)

24-h mortality (yes)

Hospital LOS, median days (IQR)

Total prehospital time,

median min. (IQR)

0.077

0.611

$191(68.5) \quad 74(71.2)$

$88(31.5) \quad 30(28.8)$

0.105

Prehospital on-scene time, median min. (IQR) 
Table 3 Blunt trauma, injury mechanism by age groups and by gender, $\mathrm{n}$ and (\%)

\begin{tabular}{|c|c|c|c|c|c|}
\hline \multirow[t]{2}{*}{ Gender } & \multicolumn{5}{|c|}{ Injury mechanism category } \\
\hline & Age groups & Traffic-related & Low-energy falls & High-energy falls & Other \\
\hline \multirow[t]{3}{*}{ Male } & $15-39$ & $60(53.1)$ & $3(7.1)$ & $21(31.8)$ & $14(45.2$ \\
\hline & $40-64$ & $36(31.9)$ & $11(26.2)$ & $27(40.9)$ & $16(51.6)$ \\
\hline & $=>65$ & $17(15.0)$ & $28(66.7)$ & $18(27.3)$ & $1(3.2)$ \\
\hline \multirow[t]{3}{*}{ Female } & $15-39$ & $17(44.7)$ & $2(7.4)$ & $12(44.4)$ & $4(66.7)$ \\
\hline & $40-64$ & $12(31.6)$ & $4(14.8)$ & $8(29.6)$ & $2(33.3)$ \\
\hline & $=>65$ & $9(23.7)$ & $21(77.8)$ & $7(25.9)$ & $0(0.0)$ \\
\hline
\end{tabular}

the age group 15-39 years (OR, 2.11; $95 \%$ CI, 1.02-4.37; $p=0.044$ ) to receive analgesics. The analysis also revealed a lesser likelihood for patients with a systolic blood pressure below $90 \mathrm{mmHg}$ to receive prehospital analgesics (OR, 0.4; $95 \% \mathrm{CI}, 0.17-0.87 ; p=0.022$ ), as well as a lesser likelihood of receiving analgesics if the injury mechanism was a low-energy fall (OR, 0.15; $95 \% \mathrm{CI}$, 0.04-0.66). No interactions were found between gender and age group 15-39 years $(p=0.728)$, systolic blood pressure below $90 \mathrm{mmHg}(p=0.891)$, or a low-energy fall $(p=0.732)$.

\section{Discussion}

The aim of this study was to explore gender-related differences in prehospital trauma care of severely injured trauma patients with a special focus on triage, transportation, and prehospital interventions. The main finding was that female trauma patients were less likely to be given the highest prehospital priority, the highest prehospital competence level, and direct transport to the designated trauma center. We did not, however, find any differences between the genders regarding administered prehospital interventions, LOS at hospital, or other outcomes.

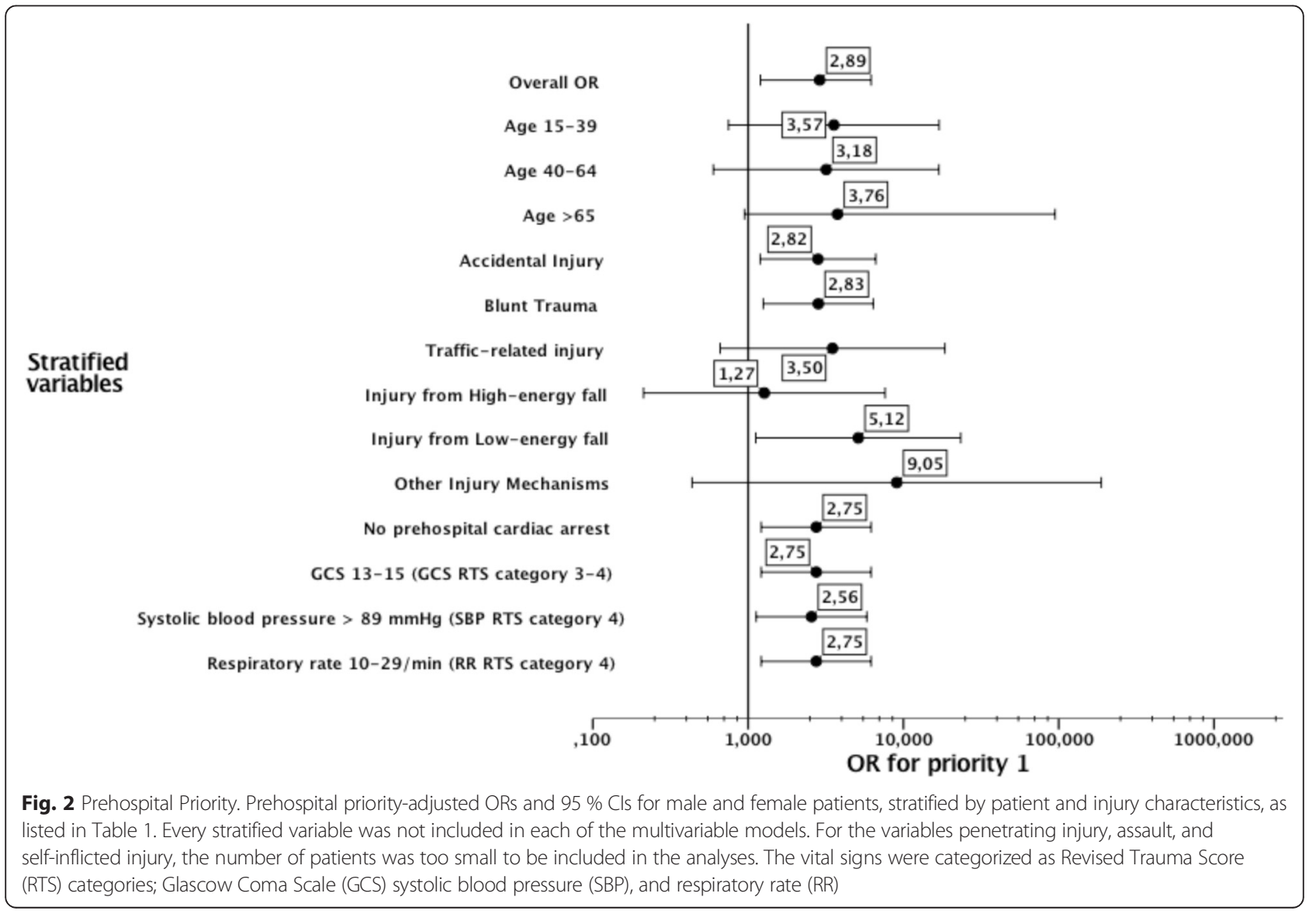


Our results point towards a gender-related difference in prehospital assessments of the severity and handling of trauma patients, which is in line with a study by Chang et al. [21] focusing on undertriage in an elderly population ( $>65$ years). In a subanalysis of their main work, they also reported on transport to a designated trauma center of priority- 1 patients who met the ACS criteria. The results of this subanalysis showed that males were significantly more likely (OR 1.36) to be transported to a trauma center than females. The authors did not further explore this finding, but it is of interest since their population consisted of individuals over 65 years of age. Hsia et al. [22] demonstrated a similar finding to that of Chang et al. in their study on trauma center use among elderly patients and showed that female gender entailed a lesser likelihood of being admitted to a trauma center. Gomez et al. [8] also demonstrated a lower likelihood for females to be admitted to a trauma center after adjusting for other factors, such as age, injury severity, type of prehospital provider, and mechanism of injury. These studies, despite different aims, suggest that also in an elderly population, males are more likely to be transported to a trauma center.

We found differences in trauma mechanism between genders, namely, that the second most common trauma mechanism for females was a low-energy fall (26.9 \%). On stratifying for age and trauma mechanism within the dominant blunt trauma group, the difference was even more pronounced (Table 3). Gomez et al. [8] reported a similar pattern and showed that falls from the same level constituted $41 \%$ of the trauma cases among females in their population, which was also the most common trauma mechanism in their study regardless of age. Perhaps this might be one of the reasons why females, despite severe injury, are not recognized at scene as potential severe trauma patients since the trauma mechanism is considered to be of low energy. In our study, we adjusted for age and trauma mechanism, but still males were more likely to be prioritized higher. However, this might only apply to female patients with normal physiological parameters in cases where the triage protocol does not consider the potential difference between genders in symptom presentation. If the trauma mechanism seems mild, the need for a trauma center transport might not be obvious. This could be one of the reasons for the difference between genders in our study.

Earlier studies focusing on the association between gender and trauma mortality are inconclusive [23]. It has been suggested that different biological features of males and females might impact trauma survival. Some studies argue that estrogens are protective in terms of survival after trauma-related shock. Haider et al. showed that females in the fertile period of life had a $14 \%$ lesser risk of dying from trauma-related shock compared to males [24]. This difference has not been seen when comparing males with pre- and post-hormonal females. Male gender has been shown to be a risk factor for one-year mortality, but not for 30-day mortality in elderly populations [6], while other studies have shown no differences in mortality between genders $[25,26]$. We did not have the data for a one-year follow-up, but for 24-h and 30-day mortality, no differences between genders were noted.

The importance of a short on-scene time has been discussed in several studies. Some have reported that helicopter transport and/or intubation might prolong the on-scene times $[27,28]$ and also the presence of a physician on scene [29]. On the other hand, the latter has also been associated with a more agressive treatment, high-precision triage, and rapid transport to the correct level of care [30]. In this study, we chose to focus on the association between on-scene time and gender and found no differences. However, a significant difference between genders was demonstrated regarding the competence level of the prehospital staff or advanced life support provided on scene (i.e., presence of a nurse anesthetist or anesthesiologist), a finding that we have not found in any other published reports. It is not obvious what these findings represent, and they need further investigation.

Trauma occurs more frequently among males $[5,23$, $25,31,32$ ], a fact confirmed by this study: $72.8 \%$ of the patients in our study were males. The predominant injury mechanism was traffic-related, which conforms with the fact, that in Sweden, severe trauma is most frequently related to motor-vehicle crashes [33, 34]. Annually, an average of 7100 males (61 \%) and 4600 females (39 \%) are hospitalized due to motor-vehicle crashes [33]. In 2012, 218 males (76 \%) and 67 females (24\%) in Sweden died in motor-vehicle crashes [34], showing a gender difference in mortality rates which is consistent worldwide [11].

On evaluating the on-scene variables, i.e., airway management, administration of intravenous fluids, pain management, and stabilization of neck and spine, no gender differences were evident in our data, which is well in line with the study by Schoeneberg et al. [23]. Other studies from the prehospital settings have shown that females reported more pain, but were less likely to receive morphine [35] and that female patients with isolated extremity injuries were less likely to receive analgesics [36]. On the other hand, Raftery et al. [37] investigated patients in the ED presenting with headache, neck pain, or back pain and concluded that females were more likely than males to report pain and also to receive more analgesics.

Our triage protocol and transport directives state that if the patient is recognized as priority 1 , the patient should be transported directly to the trauma center, but 
in cases where a patient has a compromised airway, the EMS are allowed to choose a closer non-trauma hospital to secure the airway. This aspect has not been included in our analysis. It is also worth noticing that in the current trauma triage protocol, age is not included in the triage algorithm.

A strengt of this study is that we included all patients during one year in a well-defined area covering about one fifth of Sweden's population and all patients were traceable via the unique individual Swedish social security number. Nevertheless, the relatively small study sample and the fact that the partient-related outcome was measured only in terms of mortality may limit the validity of our findings.

\section{Conclusions}

In an urban part of Sweden covering one fifth of the Swedish population, we found that female trauma patients were less likely to receive the highest prehospital transport priority and were less likely to be transported directly from the scene to a trauma center. We also found that the trauma mechanism differed between genders, but this did not affect the outcome. Prehospital interventions and other system outcomes did not differ between genders. Recognizing gender differences with educational efforts and in pre-hospital trauma management protocols may expedite the trauma care of female patients.

\section{Abbreviations \\ ACOS: American College of Surgeons; AIS: Abbreviated Injury Scale; CPR: Cardiopulmonary resuscitation; ED: Emergency Department; EMS: Emergency Medical Services; EMT: Emergency medical technician; GCS: Glasgow Coma Scale; ICU: Intensive care unit; ISS: Injury Severity Score; KSS: Karolinska University Hospital in Solna; KVITTRA/QUITC: (Quality in Trauma Care); LOS: Length of stay (days); ROSC: Return of spontaneous circulation; RTS: Revised Trauma Score; RR: Respiratory rate; SBP: Systolic blood pressure; SCC: Stockholm County Council.}

\section{Competing interests}

The authors declare that they have no competing interests.

\section{Authors' contributions}

RRW conceived the study, participated in the design of the study and coordination, collected the data, performed the statistical analysis, and drafted the manuscript. SP Participated in the design of the study, provided methodological and statistical support, and helped to draft the manuscript. $\mathrm{HL}$ participated in the analyses and in drafting the manuscript. MS participated in the design of the study and helped in drafting the manuscript. HM provided support in the design and helped in drafting the manuscript. MC conceived the study, participated in its design and helped in drafting the manuscript. All authors read and approved the final manuscript.

\section{Acknowledgments}

We thank Hans Järnbert Petterson, PhD and statistician at Karolinska Institutet, Department of Clinical Science and Education, Södersjukhuset, for his support in the statistical analyses, and Milka Dinevik, controller at the ambulance service company, AISAB (Ambulanssjukvården i Storstockholm AB) for helping in collecting data. This study was supported by grants provided by the Stockholm County Council (ALF Project).

\section{Author details}

'Department of Clinical Science and Education, Södersjukhuset, Karolinska Institutet, SE-118 83 Stockholm, Sweden. ${ }^{2}$ Division of Intensive Care
Medicine, Department of Anesthesiology, Intensive Care and Pain Medicine, University of Helsinki and Helsinki University Hospital, Helsinki, Finland. ${ }^{3}$ Field of Prehospital Critical Care, Network for Medical Sciences, University of Stavanger, Kjell Arholmsgate 41, NO-4036 Stavanger, Norway. ${ }^{4}$ Department of Emergency Medicine and Services, University of Helsinki and Helsinki University Hospital, Helsinki, Finland. ${ }^{5}$ Department of Anesthesia and Intensive Care, Södersjukhuset, SE-118 83 Stockholm, Sweden.

Received: 25 May 2015 Accepted: 6 January 2016

Published online: 19 January 2016

\section{References}

1. Holder Y. Injury Surveillance Guidelines. Geneva: World Health Organization; 2002.

2. Gedeborg R, Chen LH, Thiblin I, Byberg L, Melhus H, Michaelsson K, et al. Prehospital injury deaths - strengthening the case for prevention: nationwide cohort study. J Trauma Acute Care Surg. 2012;72(3):765-72.

3. Rossaint R, Bouillon B, Cerny V, Coats TJ, Duranteau J, Fernandez-Mondejar E, et al. Management of bleeding following major trauma: an updated European guideline. Crit Care. 2010;14(2):R52.

4. Gaarder C, Naess PA, Frischknecht Christensen E, Hakala P, Handolin L, Heier HE, et al. Scandinavian Guidelines - "The massively bleeding patient". Scand J Surg. 2008:97(1):15-36.

5. Wohltmann CD, Franklin GA, Boaz PW, Luchette FA, Kearney PA, Richardson $J D$, et al. A multicenter evaluation of whether gender dimorphism affects survival after trauma. Am J Surg. 2001;181(4):297-300.

6. Brattstrom O, Larsson E, Granath F, Riddez L, Bell M, Oldner A. Time dependent influence of host factors on outcome after trauma. Eur $J$ Epidemiol. 2012;27(3):233-41.

7. Hesselfeldt R, Steinmetz J, Jans H, Jacobsson MB, Andersen DL, Buggeskov $K$, et al. Impact of a physician staffed helicopter on a regional trauma system: a prospective, controlled, observational study. Acta Anaesthesiol Scand. 2013;57(5):660-8.

8. Gomez D, Haas B, de Mestral C, Sharma S, Hsiao M, Zagorski B, et al. Gender-associated differences in access to trauma center care: a population-based analysis. Surgery. 2012;152(2):179-85.

9. Meisel ZF, Armstrong K, Mechem CC, Shofer FS, Peacock N, Facenda K, et al. Influence of sex on the out-of-hospital management of chest pain. Acad Emerg Med. 2010;17(1):80-7.

10. Kaul P, Chang WC, Westerhout CM, Graham MM, Armstrong PW. Differences in admission rates and outcomes between men and women presenting to emergency departments with coronary syndromes. Can Med Assoc J. 2007;177(10):1193-9.

11. World Health O. World Health Report 2002: Reducing Risks. Geneva: Promoting Healthy Life; 2002.

12. Equal Care?: a gender perspective on health care [in swedish] Jämställd vård?: könsperspektiv på hälso- och sjukvården. Stockholm: Socialstyrelsen; 2004.

13. Stockholms läns landsting. http://www.sll.se (2013). Accessed 1 Oct 2013.

14. Trauma programs. http://www.facs.org/trauma/ (2013). Accessed 30 Oct 2013.

15. Stockholms läns landsting. Medicinska riktlinjer. 2012 www.aisab.nu/ media/49863/2015_medicinska_behandlingsriktlinjer.pdf. Accessed 22 March 2012.

16. American College of Surgeons: Trauma Programs. http://www.facs.org/trauma/ (2013). Accessed 30 Oct 2013

17. Ringdal KG, Coats TJ, Lefering R, Di Bartolomeo S, Steen PA, Roise O, et al. The Utstein template for uniform reporting of data following major trauma: a joint revision by SCANTEM, TARN, DGU-TR and RITG. Scand I Trauma Resusc Emerg Med. 2008;16:7.

18. Osler T, Baker SP, Long W. A modification of the injury severity score that both improves accuracy and simplifies scoring. J Trauma. 1997;43(6):922-5. discussion 925-926.

19. Schluter PJ. The Trauma and Injury Severity Score (TRISS) revised. Injury. 2011:42(1):90-6.

20. Teasdale G, Jennett B. Assessment of coma and impaired consciousness. A practical scale. Lancet. 1974;2(7872):81.

21. Chang DC, Bass RR, Cornwell EE, Mackenzie EJ. Undertriage of elderly trauma patients to state-designated trauma centers. Arch Surg. 008;143(8):776-81. discussion 782. 
22. Hsia RY, Wang E, Saynina O, Wise P, Perez-Stable EJ, Auerbach A. Factors associated with trauma center use for elderly patients with trauma: a statewide analysis, 1999-2008. Arch Surg. 2011;146(5):585-92.

23. Schoeneberg C, Kauther MD, Hussmann B, Keitel J, Schmitz D, Lendemans S. Gender-specific differences in severely injured patients between 2002 and 2011: data analysis with matched-pair analysis. Crit Care. 2013;17(6):R277.

24. Haider AH, Crompton JG, Chang DC, Efron DT, Haut ER, Handly N, et al. Evidence of hormonal basis for improved survival among females with trauma-associated shock: an analysis of the National Trauma Data Bank. J Trauma. 2010;69(3):537-40.

25. Gannon CJ, Napolitano LM, Pasquale M, Tracy JK, McCarter RJ. A statewide population-based study of gender differences in trauma: validation of a prior single-institution study. J Am Coll Surg. 2002;195(1):11-8.

26. Croce MA, Fabian TC, Malhotra AK, Bee TK, Miller PR. Does gender difference influence outcome? J Trauma. 2002;53(5):889-94.

27. Wyen H, Lefering R, Maegele M, Brockamp T, Wafaisade A, Wutzler S, et al. The golden hour of shock - how time is running out: prehospital time intervals in Germany - a multivariate analysis of 15,103 patients from the Trauma Register DGU(R). Emerg Med J. 2013;30(12):1048-55.

28. Ringburg AN, Spanjersberg WR, Frankema SP, Steyerberg EW, Patka P, Schipper IB. Helicopter emergency medical services (HEMS): impact on on-scene times. J Trauma. 2007;63(2):258-62.

29. Klemen P, Grmec S. Effect of pre-hospital advanced life support with rapid sequence intubation on outcome of severe traumatic brain injury. Acta Anaesthesiol Scand. 2006;50(10):1250-4.

30. lirola TT, Laaksonen Ml, Vahlberg TJ, Pälve HK. Effect of physician-staffed helicopter emergency medical service on blunt trauma patient survival and prehospital care. Eur J Emerg Med. 2006;13(6):335-9.

31. Magnotti LJ, Fischer PE, Zarzaur BL, Fabian TC, Croce MA. Impact of gender on outcomes after blunt injury: a definitive analysis of more than 36,000 trauma patients. J Am Coll Surg. 2008;206(5):984-91. discussion 991-982.

32. Fowler RA, Sabur N, Li P, Juurlink DN, Pinto R, Hladunewich MA, et al. Sex- and age-based differences in the delivery and outcomes of critical care. Can Med Assoc J. 2007;177(12):1513.

33. The public healh in Sweden - yearly report 2013 [in Swedish]. Folkhälsan i Sverige - Årsrapport 2013. Stockholm: Socialstyrelsen 2014

34. Road traffic injuries 2012 [in Swedish]. Vägtrafikskador 2012 Stockholm: Trafikanalys; 2013

35. Lord B, Cui J, Kelly AM. The impact of patient sex on paramedic pain management in the prehospital setting. Am J Emerg Med. 2009;27(5):525-9.

36. Michael GE, Sporer KA, Youngblood GM. Women are less likely than men to receive prehospital analgesia for isolated extremity injuries. Am J Emerg Med. 2007;25(8):901-6

37. Raftery KA, Smith-Coggins R, Chen AH. Gender-associated differences in emergency department pain management. Ann Emerg Med. 1995;26(4):414-21.

\section{Submit your next manuscript to BioMed Central and we will help you at every step:}

- We accept pre-submission inquiries

- Our selector tool helps you to find the most relevant journal

- We provide round the clock customer support

- Convenient online submission

- Thorough peer review

- Inclusion in PubMed and all major indexing services

- Maximum visibility for your research

Submit your manuscript at www.biomedcentral.com/submit
Biomed Central 